\title{
Discrete Solution of the Plateau Problem and Its Convergence
}

\author{
By Takuya Tsuchiya*
}

\begin{abstract}
In this paper we define a discrete solution of the Plateau problem and we prove theorems for the convergence of the discrete solution when the contour satisfies a certain condition. A numerical example is given.
\end{abstract}

1. Introduction. Let $D=\left\{(u, v) \in \mathbf{R}^{2} \mid u^{2}+v^{2}<1\right\}$ be the unit disk with boundary $\partial D$, and let $\Gamma$ be a Jordan curve in $n$-dimensional Euclidean space $\mathbf{R}^{n}$, $n \geqslant 2$, that is, $\Gamma$ is a topological image of $\partial D$. The Plateau problem is to find maps $x(u, v)=\left(x_{1}(u, v), \ldots, x_{n}(u, v)\right)$ from the closure $\bar{D}$ of $D$ into $\mathbf{R}^{n}$ which have the following properties:

(1) Each $x_{i}$ is continuous on $\bar{D}$ and harmonic in $D$.

(2) The parameters $u, v$ are isothermal in $D$, that is,

$$
\left|x_{u}\right|^{2}=\left|x_{v}\right|^{2}, \quad\left(x_{u}, x_{v}\right)=0
$$

where $x_{u}=\left(\partial x_{1} / \partial u, \ldots, \partial x_{n} / \partial u\right)$ and $x_{v}=\left(\partial x_{1} / \partial v, \ldots, \partial x_{n} / \partial v\right)$.

(3) The restriction of $x$ to $\partial D$ is a homeomorphism between $\partial D$ and $\Gamma$, in which three fixed points on $\partial D$ correspond to three fixed points on $\Gamma$.

The notations $|\cdot|$ and $(\cdot, \cdot)$ mean Euclidean norm and inner product of $\mathbf{R}^{n}$, respectively.

A solution of the Plateau problem is called a minimal surface spanned in $\Gamma$. In [9] and [10] numerical methods for approximating minimal surfaces were given. The purpose of this paper is to demonstrate that their methods are proper, that is, these numerical solutions converge to minimal surfaces in suitable function spaces.

It is known that the Plateau problem is equivalent to the following variational problem.

Let $C\left(\bar{D} ; \mathbf{R}^{n}\right)$ be the space of continuous maps on $\bar{D}$, and let $H^{1}\left(D ; \mathbf{R}^{n}\right)$ be the ordinary Sobolev space (the exact definitions will be given in Section 2). We define the class $X_{\Gamma}$ of maps by

$$
X_{\Gamma}=\left\{f \in C\left(\bar{D} ; \mathbf{R}^{n}\right) \cap H^{1}\left(D ; \mathbf{R}^{n}\right)|f(\partial D)=\Gamma, f|_{\partial D} \text { is monotone }\right\}
$$

Received February 7, 1985; revised May 23, 1985 and November 11, 1986.

1980 Mathematics Subject Classification (1985 Revision). Primary 49F10, 65E05, 65N99.

* This research is partially supported by Grant-in-Aid for Encouragement of Young Scientists No. 60740119, the Ministry of Education. 
where monotone means that, for each $p \in \Gamma,\left(\left.f\right|_{\partial D}\right)^{-1}(p) \subset \partial D$ is connected. $X_{\Gamma}$ may be empty [6, p. 58], but in this paper we assume that $X_{\Gamma} \neq \varnothing$. We choose six arbitrary distinct points $z_{1}, z_{2}, z_{3} \in \partial D$ and $\zeta_{1}, \zeta_{2}, \zeta_{3} \in \Gamma$, and we define the subset of $X_{\Gamma}$ by

$$
X_{\Gamma}^{t p}=\left\{f \in X_{\Gamma} \mid f\left(z_{i}\right)=\zeta_{i}, i=1,2,3\right\},
$$

where the superscript " $t p$ " stands for "three-point condition". For $f \in H^{1}\left(D ; \mathbf{R}^{n}\right)$, the energy of $f$ on $D$ is defined by

$$
E(f)=\frac{1}{2} \iint_{D}\left(\left|f_{u}\right|^{2}+\left|f_{v}\right|^{2}\right) d u d v .
$$

$E$ is called the energy functional. It is well known that $f \in X_{\Gamma}^{t p}$ is a minimal surface spanned in $\Gamma$ if and only if $f$ is a stationary point of the energy functional in $X_{\Gamma}^{t p}$. In particular, if $f$ is a minimal point of $E$ in $X_{\Gamma}^{t p}$, then $f$ is a minimal surface spanned in $\Gamma[4$, pp. 107-118].

In Section 3 we define a (stable) discrete minimal surface by a variational method using the simplest finite element scheme. In Section 5 we prove the convergence of the discrete minimal surfaces.

For the existence of minimal surfaces, Douglas and Rado proved the following theorem ([4, pp. 101-105], [6, p. 71]):

TheOREM A. Let $e_{\Gamma}=\inf \left\{E(f): f \in X_{\Gamma}^{t p}\right\}$. If $X_{\Gamma}^{t p} \neq \varnothing$, then there exists a map $x \in X_{\Gamma}^{t p}$ such that $E(x)=e_{\Gamma}$.

From the above facts, $x \in X_{\Gamma}^{t p}$ in Theorem A is a minimal surface spanned in $\Gamma$. Hence we can say that if $X_{\Gamma}^{t p} \neq \varnothing$, there exists at least one minimal surface in $X_{\Gamma}^{t p}$. An $x$ as in Theorem A is called a Douglas solution. Note that if $n=2$, Theorem A is the Riemann Mapping Theorem for domains bounded by Jordan curves. To prove Theorem A, the following important lemma was introduced:

LEMMA B. Let $M$ be a positive constant such that $e_{\Gamma}<M$, and let $Y$ be the subset of $X_{\Gamma}^{t p}$ defined by $Y=\left\{f \in X_{\Gamma}^{t p} \mid E(f) \leqslant M\right\}$. Then $Y$ is equicontinuous on $\partial D$.

In Section 4 we prove a lemma which is the discrete analogue of Lemma B. That lemma will play an important role in the proof of the convergence of discrete minimal surfaces.

2. Function Spaces. In this section we define the function spaces to be used. Let $A$ be a compact subset of $\mathbf{R}^{2}$. We define

$$
C(A)=\{f: A \rightarrow \mathbf{R} \mid f \text { is continuous on } A\},
$$

with $\|f\|_{C(A)}=\max \{|f(u, v)|:(u, v) \in A\}$, and

$$
C\left(A ; \mathbf{R}^{n}\right)=\left\{f=\left(f_{1}, \ldots, f_{n}\right): A \rightarrow \mathbf{R}^{n} \mid f_{i} \in C(A), i=1, \ldots, n\right\},
$$

with $\|f\|_{C\left(A: \mathbf{R}^{n}\right)}=\max \left\{\left\|f_{i}\right\|_{C(A)}: i=1, \ldots, n\right\}$.

For $1 \leqslant p \leqslant \infty$, we define

$$
L^{p}\left(A ; \mathbf{R}^{n}\right)=\left\{f=\left(f_{1}, \ldots, f_{n}\right): A \rightarrow \mathbf{R}^{n} \mid f_{i} \in L^{p}(A), i=1, \ldots, n\right\},
$$

with $\|f\|_{L^{\prime \prime}\left(A: \mathbf{R}^{n}\right)}=\max \left\{\left\|f_{i}\right\|_{L^{p}(A)}: i=1, \ldots, n\right\}$. 
Let $B$ be a bounded domain in $\mathbf{R}^{2}$. We define

$$
W^{1, p}(B)=\left\{f \in L^{p}(B) \mid f_{u}, f_{v} \in L^{p}(B)\right\},
$$

with $\|f\|_{W^{1 . p}(B)}=\|f\|_{L^{p}(B)}+\left\|f_{u}\right\|_{L^{p}(B)}+\left\|f_{v^{\prime}}\right\|_{L^{p}(B)}$, where $f_{u}$ and $f_{v}$, are distributional derivatives. We define

$$
W^{1, p}\left(B ; \mathbf{R}^{n}\right)=\left\{f=\left(f_{1}, \ldots, f_{n}\right): B \rightarrow \mathbf{R}^{n} \mid f_{i} \in W^{1, p}(B), i=1, \ldots, n\right\},
$$

with $\|f\|_{W^{1, p}\left(B: \mathbf{R}^{n}\right)}=\max \left\{\left\|f_{i}\right\|_{W^{1, p}(B)}: i=1, \ldots, n\right\}$. When $p=2$, we denote $W^{1, p}(B)$ and $W^{1, p}\left(B ; \mathbf{R}^{n}\right)$ by $H^{1}(B)$ and $H^{1}\left(B ; \mathbf{R}^{n}\right)$, respectively. We define

$$
H M=\left\{f=\left(f_{1}, \ldots, f_{n}\right): D \rightarrow \mathbf{R}^{n} \mid f_{i} \text { is harmonic in } D, i=1, \ldots, n\right\} .
$$

An element of $H M$ is called a harmonic map.

For simplicity, when there is no confusion, we omit "; $\mathbf{R}^{n}$ " from the notations.

3. Definition of the Discrete Minimal Surface. Let $\Omega \subset D$ be a triangulation of $D$, that is, $\Omega$ is a polygonal domain with the properties that (1) $\partial \Omega$, the boundary of $\Omega$, inscribes $\partial D$, and (2) the set $\bar{\Omega}$ is the union $\bar{\Omega}=\bigcup K_{i}$ of finite triangles $\left\{K_{i}\right\}$ whose interiors are pairwise disjoint and such that all their edges are either an edge of another triangle or an edge of the polygon $\partial \Omega$.

With the triangulation $\Omega$ we associate the mesh size of $\Omega$ defined by

$$
|\Omega|=\max _{i} \operatorname{diam}\left(K_{i}\right) .
$$

We assume that there exists a positive constant $\omega$ which is independent of the triangulation $\Omega$ such that the following inequality holds for each triangle $K_{i} \subset \Omega$ :

$$
\operatorname{diam}\left(K_{i}\right) / \rho\left(K_{i}\right) \leqslant \omega,
$$

where $\rho\left(K_{i}\right)=\sup \left\{\operatorname{diam}(S) ; K_{i} \supset S\right.$ : ball $\}$.

Let $S_{\Omega}$ be the set of functions which are continuous on $\bar{\Omega}$ and linear on each triangle $K_{i}$. Let $\mathbb{S}_{\Omega}$ be the set of maps from $\bar{\Omega}$ into $\mathbf{R}^{n}$ such that each component function belongs to $S_{\Omega}$. Let $N_{\Omega}=\left\{b_{i}\right\}_{i=1}^{N+N^{\prime}}$ be the set of nodal points of $\Omega$ where $b_{i} \in \Omega^{\circ}$, the interior of $\Omega$, for $1 \leqslant i \leqslant N$, and $b_{i} \in \partial \Omega$ for $N+1 \leqslant i \leqslant N+N^{\prime}$. The basis $\left\{w_{i}\right\}_{i=1}^{N+N^{\prime}} \subset S_{\Omega}$ of $S_{\Omega}$ is defined by

$$
w_{i}\left(b_{j}\right)=\delta_{i j}, \quad 1 \leqslant i, j \leqslant N+N^{\prime} .
$$

We assume that the following inequality holds:

$$
\iint_{\Omega}\left(w_{i u} w_{j u}+w_{i v}, w_{j v}\right) d u d v \leqslant 0 \quad \text { for } 1 \leqslant i \leqslant N, 1 \leqslant j \leqslant N+N^{\prime}, i \neq j .
$$

We say that a triangulation $\Omega$ satisfying $(\mathrm{H} 2)$ is of nonnegative type. We associate the admissible class of triangulations of $D$ defined by

$$
\Delta^{t p}=\left\{\Omega \mid z_{1}, z_{2}, z_{3} \in N_{\Omega}, \Omega \text { satisfies }(\mathrm{H} 1),(\mathrm{H} 2)\right\} .
$$

When $\Omega$ is given, we define

$$
X_{\Gamma, \Omega}=\left\{f \in \mathbb{S}_{\Omega}\left|f\left(N_{\Omega} \cap \partial D\right) \subset \Gamma, f\right|_{\partial D} \text { is } d \text {-monotone }\right\},
$$

where $d$-monotone means that the order of nodal points on $\Gamma$ is the same as the order of nodal points on $\partial D$. Let

$$
X_{\Gamma, \Omega}^{t p}=\left\{f \in X_{\Gamma, \Omega} \mid f\left(z_{i}\right)=\zeta_{i}, i=1,2,3\right\},
$$


and let $E_{\Omega}(f)$ be the energy functional on $\Omega$ defined by

$$
E_{\Omega}(f)=\frac{1}{2} \iint_{\Omega}\left(\left|f_{u}\right|^{2}+\left|f_{v}\right|^{2}\right) d u d v .
$$

We extend $f \in \mathbb{S}_{\Omega}$ to $D-\Omega$ as follows:

If $p \in \partial \Omega$ and $p \notin N_{\Omega}$, there exists an exterior normal half line $L_{p}$ of $\partial \Omega$ on $p$. For arbitrary $q \in L_{p} \cap(D-\Omega)$, we define $f(q)=f(p)$. Then the following estimate is valid:

$$
E_{\Omega}(f) \leqslant E(f) \leqslant(1+C|\Omega|) E_{\Omega}(f) \text { for any } f \in \mathbb{S}_{\Omega}
$$

where $C$ is a constant which is independent of $\Omega$ and $f$.

Definition 1. Let $\Omega \in \Delta^{t p}$.

(D1) $f \in S_{\Omega}$ is a d-harmonic function on $\Omega$ if $E_{\Omega}(f) \leqslant E_{\Omega}(g)$ for any $g \in S_{\Omega}$ with $g=f$ on $\partial \Omega$.

(D2) $f=\left(f_{1}, \ldots, f_{n}\right) \in \mathbb{S}_{\Omega}$ is a $d$-harmonic map on $\Omega$ if $f_{i}, i=1, \ldots, n$, are $d$-harmonic functions. We denote by $H M_{\Omega}$ the set of $d$-harmonic maps on $\Omega$.

(D3) Let $x \in H M \cap C\left(\bar{D} ; \mathbf{R}^{n}\right) . f \in \mathbb{S}_{\Omega}$ is the FEM solution of $x$ on $\Omega$ if $f \in H M_{\Omega}$ and $x(b)=f(b)$ for all $b \in N_{\Omega} \cap \partial D$.

(D4) $f \in X_{\Gamma, \Omega}^{t p}$ is a stable d-minimal surface if there exists a positive constant $\delta$ such that

$$
\|f-g\|_{C(\bar{\Omega})}<\delta \quad \text { implies } \quad E_{\Omega}(f) \leqslant E_{\Omega}(g) \text { for } g \in X_{\Gamma, \Omega}^{t p} .
$$

(D5) $f \in X_{\Gamma, \Omega}^{t p}$ is a $d$-Douglas solution if

$$
E_{\Omega}(f)=\inf \left\{E_{\Omega}(g): g \in X_{\Gamma, \Omega}^{t p}\right\} .
$$

From these definitions it is obvious that a stable $d$-minimal surface is $d$-harmonic, and a $d$-Douglas solution is a stable $d$-minimal surface.

Remark 2. It is known that there exists an unstable minimal surface in $X_{\Gamma}^{t p}$ if there exist two stable minimal surfaces in $X_{\Gamma}^{t p}$ [4, pp. 236-243]. We can define the unstable d-minimal surface similarly as in Definition 1 . But a proof of the convergence of unstable $d$-minimal surfaces has not yet been obtained. So we do not examine unstable $d$-minimal surfaces in this paper.

Lemma 3. Suppose that $\Omega \in \Delta^{t p}$. Then a d-harmonic function $f$ satisfies the discrete maximum principle,

$$
\min _{s \in \partial D} f(s) \leqslant f(z) \leqslant \max _{s \in \partial D} f(s) \quad \text { for any } z \in D .
$$

Proof. We can prove this lemma by direct computation. See [1], [3].

4. Relative Compactness. In this section we prove the relative compactness of bounded subsets of $X_{\Gamma, \Omega}^{t p}$ when the Jordan curve satisfies a certain condition. This fact is the discrete analogue of Lemma $B$.

Let $\Gamma_{0} \subset \mathbf{R}^{n}$ be a Jordan arc which connects two distinct points $a, b \in \mathbf{R}^{n}$, and let $c \neq a, b$ be a point on $\Gamma_{0}$. Taking finite points $a=x_{0}, x_{1}, \ldots, x_{l}=c, \ldots, x_{m}=b$ monotonously on $\Gamma_{0}$, and connecting $x_{i-1}$ and $x_{i}, i=1, \ldots, m$, by segments, a continuous and piecewise linear arc is defined. We denote by $L_{\Gamma_{0}, c}$ the set of such arcs. 
Let $\Gamma \subset \mathbf{R}^{n}$ be a Jordan curve and $\zeta_{i}(i=1,2,3)$ be three distinct points on $\Gamma$.

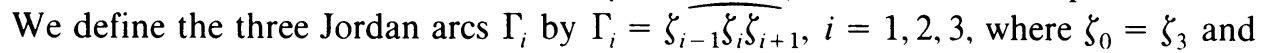
$\zeta_{4}=\zeta_{1}$.

We say that $\Gamma=\left\{\Gamma, \zeta_{1}, \zeta_{2}, \zeta_{3}\right\}$ has the property (A) if $\Gamma$ satisfies the following condition:

(A) For an arbitrary positive number $\varepsilon$ there exists a positive number $\sigma$ such that for any $\eta \in L_{\Gamma_{1}, \zeta}, i=1,2,3$, and any $p_{1}, p_{2} \in \eta-\left\{\zeta_{i-1}, \zeta_{i+1}\right\}$ with $\left|p_{1}-p_{2}\right| \geqslant \varepsilon$, $\eta-\left\{p_{1}, p_{2}\right\}$ has three connected components $\eta_{i}, i=1,2,3$, with $\zeta_{i-1} \in \eta_{1}$ and $\zeta_{i+1} \in \eta_{3}$, and the distance between $\eta_{1}$ and $\eta_{3}$ is greater then $\sigma$, i.e.,

$$
\operatorname{dist}\left(\eta_{1}, \eta_{3}\right) \geqslant \sigma \text {. }
$$

The condition (A) is very restrictive. This condition requires that $\Gamma$ should be almost like a circle. A contour like in Figure 1 does not satisfy (A).

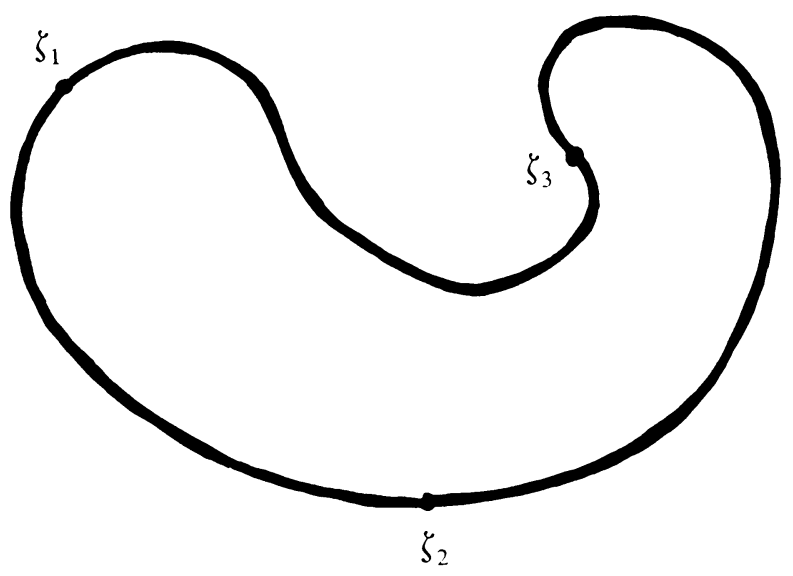

FIGURE 1

But if $n=2$, the condition (A) does not restrict us. See Remark 10.

Let $M$ be a positive constant with $e_{\Gamma}<M$. We define

$$
Y^{\prime}=\left\{f \in X_{\Gamma, \Omega}^{t p} \mid \Omega \in \Delta^{t p}, E(f) \leqslant M\right\} .
$$

Lemma 4. Suppose that $\left\{\Gamma, \zeta_{1}, \zeta_{2}, \zeta_{3}\right\}$ satisfies (A). Then $Y^{\prime}$ is equicontinuous on $\partial D$.

Proof. For arbitrary $z \in \mathbf{R}^{2}$ and arbitrary positive number $r$, we define

$$
C_{r, z}=D \cap\left\{w \in \mathbf{R}^{2}:|w-z|=r\right\} .
$$

Let $f \in Y^{\prime}$, and let $l\left(C_{r, z}\right)$ be the length of the image $f\left(C_{r . z}\right)$. The following lemma is valid ([4, p. 102], [6, pp. 67-68]):

Lemma 5. For arbitrary $\delta, 0<\delta<1$, there exists $\rho, \delta \leqslant \rho \leqslant \delta^{1 / 2}$, depending on $f$ and $z$ such that

$$
l\left(C_{\rho, z}\right)^{2} \leqslant 2 \pi \lambda(\delta)
$$

where $\lambda(\delta)=4 M / \log (1 / \delta)$. 
The proof of Lemma 4 is by contradiction. Assume that $Y^{\prime}$ is not equicontinuous on $\partial D$. Then there exists a positive number $\varepsilon_{0}$ such that for arbitrary $\delta>0$ there exist $\Omega \in \Delta^{t p}, f \in X_{\Gamma, \Omega}^{t p}$, and $a_{1}, a_{2} \in \partial D$ such that

$$
\left|a_{1}-a_{2}\right|<\delta \text { and }\left|f\left(a_{1}\right)-f\left(a_{2}\right)\right| \geqslant \varepsilon_{0} .
$$

Let $\sigma$ be the positive number associated with $\varepsilon=\varepsilon_{0}$ in (A). We choose $\delta$ such that (a) $\sigma>(2 \pi \lambda(\delta))^{1 / 2}$, and (b) $\min \left\{\left|z_{i}-z_{j}\right|: i \neq j\right\}>2 \delta^{1 / 2}$.

For such $\delta$, we take $\Omega \in \Delta^{t p}, f \in X_{\Gamma, \Omega}^{t p}$ and $a_{1}, a_{2} \in \partial D$ as in (4.5). Let $w$ be the center of the shorter arc $\widehat{a_{1} a_{2}}$. By Lemma 5 , there exists a number $\rho$ with $\delta \leqslant \rho \leqslant \delta^{1 / 2}$ such that $l\left(C_{\rho, w}\right) \leqslant(2 \pi \lambda(\delta))^{1 / 2}$. Let $b_{1}, b_{2}$ be the end points of $C_{\rho, w}$. $\partial D$ is divided into two connected components by $C_{\rho, k}$. An arc $A_{1}$ contains $w$ and, by (b), its complement $A_{2}$ contains at least two of the points $z_{i}, i=1,2,3$. Then $f\left(b_{j}\right), f\left(a_{j}\right)$ $(j=1,2)$ are on a Jordan arc which is an element of $L_{\Gamma_{i}, \zeta_{i}}$ for some $i$.

From (a),

$$
\left|f\left(b_{1}\right)-f\left(b_{2}\right)\right| \leqslant l\left(C_{\rho, w}\right) \leqslant(2 \pi \lambda(\delta))^{1 / 2}<\sigma
$$

and from (4.5)

$$
\left|f\left(a_{1}\right)-f\left(a_{2}\right)\right| \geqslant \varepsilon_{0} .
$$

Because of (A), this is the contradiction, and Lemma 4 is proved.

From Lemma 5 and the Ascoli-Arzelà theorem we immediately obtain the following

CorollaRY 6. Let $\Delta^{t p} \supset\left\{\Omega_{n}\right\}_{n=1}^{\infty}$ such that $\lim _{n \rightarrow \infty}\left|\Omega_{n}\right|=0$, and let $f_{n} \in X_{\Gamma, \Omega_{n}}^{t p}$. Suppose that $\left\{\Gamma, \zeta_{i}\right\}$ satisfies (A) and $E\left(f_{n}\right)$ are uniformly bounded. Then there exists a subsequence $\left\{f_{n_{1}}\right\}$ such that $\left.f_{n_{i}}\right|_{\partial D}$ converges uniformly to a continuous map $\varphi \in C(\partial D)$ on $\partial D$. Moreover, $\varphi(\partial D)=\Gamma$ and $\varphi$ is monotone.

5. Convergence of the Discrete Minimal Surfaces. In this section we prove the convergence of the discrete minimal surfaces.

Lemma 7. Let $\Delta^{t p} \supset\left\{\Omega_{n}\right\}_{n=1}^{\infty}$ such that $\lim _{n \rightarrow \infty}\left|\Omega_{n}\right|=0$, and let $\left\{w_{n} \in \mathbb{S}_{\Omega_{n}}\right\}_{n=1}^{\infty}$ be a sequence of d-harmonic maps.

Suppose that $\left.w_{n}\right|_{\partial D}$ converges uniformly to a continuous map $\varphi \in C(\partial D)$. Let $w$ be a harmonic map in $D$ with $w=\varphi$ on $\partial D$. Then we have

$$
E(w) \leqslant \liminf _{n \rightarrow \infty} E_{\Omega_{n}}\left(w_{n}\right) .
$$

Proof. Let $\left\{f_{n}\right\}_{n=1}^{\infty}$ be harmonic maps with $f_{n}=w_{n}$ on $\partial D$. Since $\left.f_{n}\right|_{\partial D}$ converges uniformly to $\varphi$, and in view of the lower semicontinuity of the energy functional [4, p. 11], we have $E(w) \leqslant \liminf _{n \rightarrow \infty} E\left(f_{n}\right)$. Since $E\left(f_{n}\right) \leqslant E\left(w_{n}\right)$, and by (3.7), we obtain (5.1).

THEOREM 8. Suppose that $\left\{\Gamma, \zeta_{i}\right\}$ satisfies (A). Let $\Delta^{t p} \supset\left\{\Omega_{n}\right\}_{n=1}^{\infty}$ be such that $\lim _{n \rightarrow \infty}\left|\Omega_{n}\right|=0$, and let $\left\{x_{n} \in X_{\Gamma, \Omega_{n}}^{i p}\right\}_{n=1}^{\infty}$ be a sequence of the $d$-Douglas solutions.

Then there exists a subsequence $\left\{x_{n_{1}}\right\}$ which converges to one of the Douglas solutions $x \in X_{\Gamma}^{t p}$ in the following sense:

$$
\lim _{n_{i} \rightarrow \infty}\left\|x-x_{n_{1}}\right\|_{H^{1}(D)}=0,
$$


and if $x \in W^{1, p}(D), p>2$, then

$$
\lim _{n_{i} \rightarrow \infty}\left\|x-x_{n_{i}}\right\|_{C(\bar{D})}=0 .
$$

If the Douglas solution is unique, then $x_{n}$ converges in the sense of (5.2) and (5.3).

Proof. Since $\mathbb{S}_{\Omega_{n}}$ are finite-dimensional vector spaces, and $E_{\Omega_{n}}$ is bounded from below with the property that $E_{\Omega_{n}}(f) \rightarrow \infty$ as $|f| \rightarrow \infty$ for $f \in X_{\Gamma, \Omega_{n}}^{t p}$, it is obvious that $d$-Douglas solutions exist in each $X_{\Gamma, \Omega_{n}}^{t p}$.

Let $y$ be one of the Douglas solutions and $f_{n} \in X_{\Gamma, \Omega_{n}}^{t p}$ the FEM solutions of $y$ on $\Omega_{n}$. It is well known that

$$
\lim _{n \rightarrow \infty}\left\|y-f_{n}\right\|_{H^{1}(D)}=0
$$

[2, p.134]. Since $E_{\Omega_{n}}\left(x_{n}\right) \leqslant E_{\Omega_{n}}\left(f_{n}\right),\left\{E_{\Omega_{n}}\left(x_{n}\right)\right\}$ is uniformly bounded. Thus, by Corollary 6 , there exists a subsequence which converges uniformly to a continuous map $\varphi \in C(\partial D)$ on $\partial D$ with the properties of Corollary 6. Let $x$ be a harmonic map with $x=\varphi$ on $\partial D$. From Lemma 7 we see that

$$
E(x) \leqslant \liminf _{n \rightarrow \infty} E_{\Omega_{n}}\left(x_{n}\right) \leqslant \lim _{n \rightarrow \infty} E\left(f_{n}\right)=E(y) .
$$

Hence $x$ is one of the Douglas solutions.

Let $g_{n} \in X_{\Gamma, \Omega_{n}}^{t p}$ be the FEM solutions of $x$ on $\Omega_{n}$. By Lemma 3 we have

$$
\lim _{n_{i} \rightarrow \infty}\left\|x_{n_{i}}-g_{n_{i}}\right\|_{C(\bar{D})}=0 \text {. }
$$

Hence, by (5.4), we obtain

$$
\lim _{n_{i} \rightarrow \infty}\left\|x_{n_{i}}-x\right\|_{L^{2}(D)}=0 .
$$

From (5.5), the limit of $E_{\Omega_{n}}\left(x_{n}\right)$ exists and is equal to $E(x)$. Hence, by standard Sobolev space theory, we obtain (5.2).

It is known [3] that, if $x \in W^{1, p}(D), p>2$, then

$$
\lim _{n \rightarrow \infty}\left\|x-g_{n}\right\|_{C(\bar{D})}=0 \text {. }
$$

Hence, with (5.6), we obtain (5.3).

When the Douglas solution is unique, the limit of convergent subsequences of $\left\{x_{n}\right\}$ is unique. Hence $x_{n}$ converges in the sense of (5.2) and (5.3).

A sufficient condition for $x \in W^{1, p}(D)(p>2)$ can be obtained in the following manner.

We say that the Jordan curve $\Gamma$ is of $C^{m, \alpha}$-class $(m \geqslant 1,0<\alpha<1)$ if there exists a homeomorphism $\gamma: \partial D \rightarrow \Gamma$ such that $\gamma \in C^{m, \alpha}(\partial D)$ and $\gamma^{\prime}(s)>0$ for all $s \in \partial D . C^{m, \alpha}$ denotes a Hölder space. The following theorem is known:

Theorem C (NitsChe [7]). Suppose that $\Gamma$ is of $C^{m, \alpha}$-class. Then arbitrary minimal surfaces spanned in $\Gamma$ belong to $C^{m, \alpha}(\bar{D})$.

Hence, if $\Gamma \in C^{1, \alpha}$, then arbitrary minimal surfaces spanned in $\Gamma$ belong to $W^{1, p}(D), p>2$. This condition is rough but practical enough. 
A harmonic map $x \in X_{\Gamma}^{t p}$ is said to be an isolated stable minimal surface if there exists a constant $\delta$ such that

$$
0<\|x-y\|_{C(\bar{D})}<\delta \text { implies } E(x)<E(y) \text { for } y \in X_{\Gamma}^{t p} \text {. }
$$

We have the following theorem:

THEOREM 9. Suppose that $\left\{\Gamma, \zeta_{i}\right\}$ satisfies (A). Let $\Delta^{t p} \supset\left\{\Omega_{n}\right\}_{n=1}^{\infty}$ be such that $\lim _{n \rightarrow \infty}\left|\Omega_{n}\right|=0$, and let $x \in X_{\Gamma}^{t p}$ be an isolated stable minimal surface. Then there exists a sequence $\left\{x_{n} \in X_{\Gamma, \Omega_{n}}^{t p}\right\}_{n=1}^{\infty}$ of stable d-minimal surfaces which converges to $x$ in the sense of (5.2) and (5.3).

Proof. Let $\left\{f_{n} \in X_{\Gamma, \Omega_{n}}^{t p}\right\}_{n=1}^{\infty}$ be the FEM solutions of $x$. We define $\delta$-neighborhoods of $f_{n}$ by

$$
B_{n}\left(f_{n}, \delta\right)=\left\{h_{n} \in X_{\Gamma, \Omega_{n}}^{t p} \cap H M_{\Omega_{n}} \mid\left\|f_{n}-h_{n}\right\|_{C(\bar{D})} \leqslant \delta\right\} .
$$

Since $B_{n}\left(f_{n}, \delta\right)$ is a bounded closed set in finite-dimensional Euclidean space, there exists $x_{n} \in B_{n}\left(f_{n}, \delta\right)$ such that $E_{\Omega_{n}}\left(x_{n}\right)$ attains the minimum value in $B_{n}\left(f_{n}, \delta\right)$. By (5.4), $\left\{E\left(x_{n}\right)\right\}$ is uniformly bounded. Hence there exists a subsequence $\left\{x_{n_{i}}\right\}$ which converges uniformly to a continuous map $\varphi$ on $\partial D$. Let $\psi \in X_{\Gamma}^{t p}$ be a harmonic map with $\psi=\varphi$ on $\partial D$. For arbitrary $\varepsilon>0$ we take sufficiently large $n_{i}$ such that $\left\|\left.x\right|_{\partial D}-\left.f_{n}\right|_{\partial D}\right\|_{C(\bar{D})}<\varepsilon / 2$ and $\left\|\left.\psi\right|_{\partial D}-\left.x_{i}\right|_{\partial D}\right\|_{C(\bar{D})}<\varepsilon / 2$. By (3.8), we obtain

$$
\|\psi-x\|_{C(\bar{D})}=\left\|\left.\psi\right|_{\partial D}-\left.x\right|_{\partial D}\right\|_{C(\partial D)}<\varepsilon+\left\|\left.f_{n_{i}}\right|_{\partial D}-\left.x_{n_{i}}\right|_{\partial D}\right\|_{C(\partial D)} \leqslant \varepsilon+\delta
$$

Hence we show that $\|\psi-x\|_{C(\bar{D})} \leqslant \delta$ and $E(\psi) \geqslant E(x)$. From the proof of Theorem 8 , the inverse inequality holds. Hence, by the assumption on $x$, we conclude that $x=\psi$. Again, from the proof of Theorem 8 we see that $x_{n}$ converges to $x$ in the sense of (5.2) and (5.3). It is clear that $x_{n}$ are $d$-minimal surfaces.

Remark 10. The condition (A) is strong. But if $n=2$ the condition (A) does not restrict us.

Let $D_{0} \subset \mathbf{R}^{2}$ be an arbitrary simply connected polygonal domain. We can approximate the conformal map $x^{-1}: \bar{D}_{0} \rightarrow \bar{D}$ instead of $x: \bar{D} \rightarrow \bar{D}_{0}$. Taking suitable $\zeta_{i}(i=1,2,3)$ on $\partial D,\left\{\partial D, \zeta_{i}\right\}$ satisfies (A). Thus for $x^{-1}$ we have the same conclusion as in Theorem 8 .

6. A Numerical Example. In this section we give a numerical example. Let $n=2$. We identify $\mathbf{R}^{2}$ with $C$ by the identification $z=u+i v$ and $x=x_{1}+i x_{2}$. Let $\Gamma=\{x \in C:|x|=1\}$ and $\zeta_{k}=e^{2(k-1) \pi i / 3}, k=1,2,3$. It is easy to check that $\left\{\Gamma, \zeta_{k}\right\}$ satisfies (A). We take the corresponding points $z_{1}=1, z_{2}=e^{4 \pi i / 3}$ and $z_{3}=e^{5 \pi i / 3}$. The conformal map $x: \bar{D} \rightarrow \bar{D}$ is the unique Douglas solution. The exact solution is $x=e^{4 \pi i / 3}(z-\alpha) /(\bar{\alpha} z-1)$, where $\alpha=(1+\sqrt{3} i) / 4$.

Here, we approximate this map on triangulations like in Figure 2, and we compare numerical solutions with the exact solution. This triangulation satisfies (H2).

We employ the generalized Newton method for the computations. For the details of the computations, see [9]. We give the results in Table 1. 


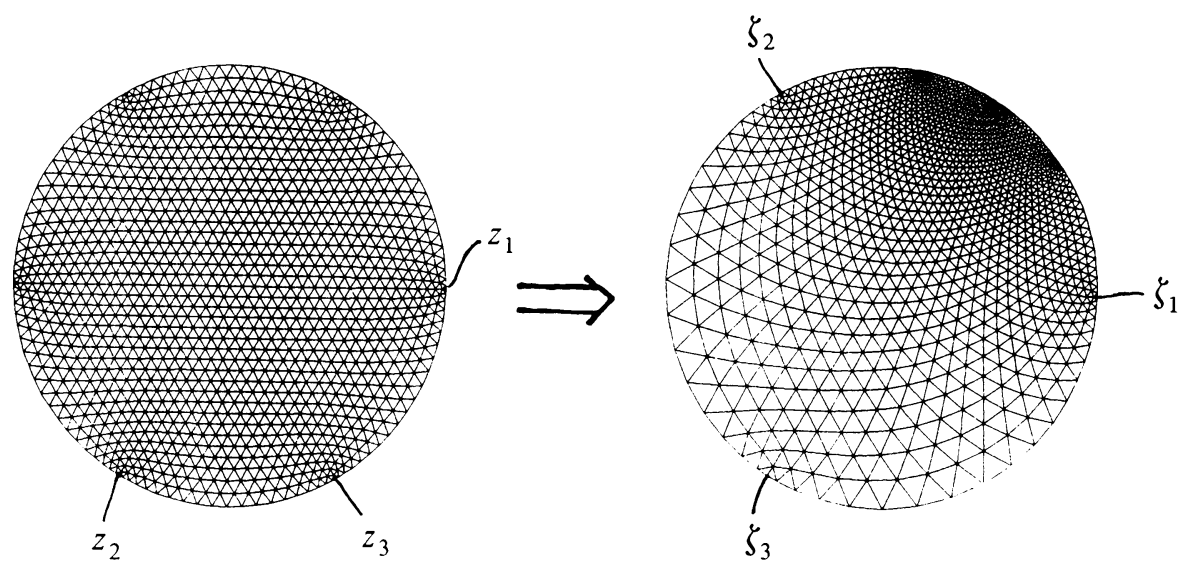

FIGURE 2

TABLE 1

Results of computations

\begin{tabular}{|c|c|c|}
\hline mesh size & norm & energy \\
\hline $1.38918 \times 10^{-1}$ & $1.25348 \times 10^{-2}$ & 3.12988 \\
\hline $9.35603 \times 10^{-2}$ & $8.77615 \times 10^{-3}$ & 3.13606 \\
\hline $7.01455 \times 10^{-2}$ & $6.57107 \times 10^{-3}$ & 3.13805 \\
\hline
\end{tabular}

In Table 1, "norm" means $\max \left\{\left|x\left(b_{i}\right)-x_{h}\left(b_{i}\right)\right|: b_{i} \in N_{\Omega}\right\}$ where $x_{h}$ is the numerical solution.

The computations were carried out using the FACOM M-382 computer system at the Computer Center, Kyushu University.

Acknowledgment. The author would like to thank Professor Seiiti Huzino and Professor Seiki Nishikawa for their helpful discussions.

Department of Mathematics

Kyushu University 33

Fukuoka 812, Japan

1. P. G. Ciarlet, "Discrete maximum principle for finite difference operators," Aequationes Math., v. 4, 1970, pp. 338-352.

2. P. G. Ciarlet, The Finite Element Method for Elliptic Problems, North-Holland, Amsterdam, 1978.

3. P. G. Ciarlet \& P. A. Raviart, "Maximum principle and uniform convergence for the finite element method," Comput. Methods Appl. Mech. Engrg., v. 2, 1973, pp. 17-31.

4. R. Courant, Dirichlet's Principle, Conformal Mapping, and Minimal Surfaces, Interscience, New York, 1950.

5. R. Courant \& D. Hilbert, Methods of Mathematical Physics, Vol. II, Interscience, New York, 1962.

6. H. B. Lawson, Jr., Lectures on Minimal Submanifolds, Vol. I, Publish or Perish, Berkeley, 1980.

7. J. C. C. Nitsche, "The boundary behavior of minimal surfaces. Kellogg's theorem and branch points on the boundary," Inventiones Math., v. 8, 1969, pp. 313-333.

8. R. Osserman, A Survey of Minimal Surfaces, Van Nostrand Reinhold, New York, 1969.

9. T. Tsuchiya, "On two methods for approximating minimal surfaces in parametric form," Math. Comp., v. 46, 1986, pp. 517-529.

10. W. L. Wil.SON, JR., "On discrete Dirichlet and Plateau problems," Numer. Math., v. 3, 1961, pp. $359-373$. 